\title{
ANALYSIS OF PATIENTS' NONADHERENCE TO STATIN THERAPY FROM CARDIOVASCULAR EVENT TO CARDIOVASCULAR REHABILITATION
}

\author{
Dragan B. Djordjević ${ }^{1,2}$, Ivan Tasić ${ }^{1,2}$, Bojana Stamenković ${ }^{1,2}$, Svetlana Kostić ${ }^{1}$, Milan Lović $^{1}$ \\ ${ }^{1}$ Institute for Prevention and Rehabilitation Niška Banja, Niš, Serbia \\ ${ }^{2}$ Faculty of Medicine, University of Niš, Niš, Serbia
}

\begin{abstract}
Numerous studies have pointed to low adherence to statin, which decreases as time period from acute cardiovascular event elapses. The aim was to analyze the cause of not taking statin by patients who were referred to rehabilitation after coronary event. Study population and methods. The research included the total of 573 patients, average age 60.3, while 305 (53.1\%) of them were patients who experienced the first cardiovascular event. The stated research was conducted by means of a questionnaire and implied active participation of the researchers in terms of monitoring the possession and use of medication during rehabilitation. On arrival to rehabilitation, 98 (17.1\%) patients did not have statin. They stated that they had never used statins before or that they stopped using them shortly after the event. This subgroup had significantly unfavorable values of lipid parameters ( $p<0.001)$, abdominal obesity ( $p<0.01)$, physical inactivity $(p<0.01)$, more comorbidities $(p<0.001)$, more prescribed medications on daily level $(p<0.05)$, lower education degree level $(p<0.01)$ and lower monthly income $(p<0.001)$. Independent factors for not taking statin were: female gender, low monthly income and large number of comorbidities $\left(R=0.291, R 2=0.85\right.$, adjusted $R^{2}=0.80$, std. error of the estimate $=0.36151 ; p<0.001)$. The patients themselves stated that the first reason for not taking statin was lack of financial funds (45.9\%), while the second reason was normalization of laboratory results (21.4\%). Three months after acute coronary event, $17.1 \%$ of patients in Serbia stopped taking statin. Lower adherence to statin closely correlates with female gender, low financial income and multiple comorbidities.
\end{abstract}

Key words: Statins, treatment, nonadherence, cardiovascular events, rehabilitation.

\section{Introduction}

The importance of statin in secondary prevention of coronary disease was proved in 1994 after the publication of 4S Study (Scandinavian Simvastatin Survival Study) which verified the reduction of mortality by $30 \%$ and coronary events by $34 \%$ [1]. After that, statin became a standard medication in secondary prevention therapy and was to be found in all manuals which refereed to secondary prevention of new cardiovascular events [2]. Observational study which was conducted in Europe showed that the percentage of prescribed medication at discharge after acute coronary event was very low, as well as that target values of lipid parameters were not in accordance with the recommendations, regardless of the fact that adequate medications were used [3]. The authors of another study have emphasized that statins were prescribed in $90 \%$ of hospital discharges and that they are now present in therapy of $84 \%$ of patients after 12 month followup [4]. When it comes to clinical practice, statins are not very welcomed by patient or even by some doctors. There are numerous prejudices which discriminate statins as

Correspondence to: Dragan Djordjević, M.D., Ph.D

Faculty of Medicine, University of Niš, Niš, Dr. 81 Dr. Zoran Đinđić Blvd., 18000 Niš, Serbia

Phone: +381648609160

E-mail: ddj964@gmail.com

Received December $13^{\text {th }}, 2017$, Accepted December $19^{\text {th }}, 2018$ "dangerous medications which destroy liver and muscles". Additionally, statin-based therapy is not always optimal and does not help in achieving targeted values of lipid parameters $[5,6]$.

Having in mind the above stated, the objective of the study was to analyze the cause of not taking statin by patients who were referred to rehabilitation after coronary event, as well as to propose measures for increasing adherence to statin therapy.

\section{Study Population and Methods}

The research included all patients with coronary events (acute myocardial infarction with or without stent implementation, coronary revascularization) who were referred to cardiovascular rehabilitation at the Institute for Treatment and Rehabilitation "Niška Banja". The patients came from all Serbian regions, except Belgrade. The stated research started in January 2013 at one of Cardiovascular Rehabilitation departments. By the end of 2016, the research included 573 patients who consecutively came to rehabilitation, $305(53.1 \%)$ of whom had one cardiovascular event, while others came after a recurrent cardiovascular event.

Research was carried out by means of a questionnaire and active participation of the researchers. A patient could answer the question by circling one of the offered answers. If the formulation of the answer was not satis- 
factory, the patient could give her/his own answer to the questions which referred to the reasons for not taking statins. Additionally, patients were free to write the primary reason for not taking statin. During the first examination, the researchers registered all medications that patients brought with them and monitored the use of medication during rehabilitation.

On examination, the weight, height and waist circumference of patients were measured. All patients were subjected to standard laboratory analysis with the aim of assessing risk factors - glycemia and lipid status (total cholesterol, HDL cholesterol, LDL cholesterol and triglycerides). Arterial blood pressure was measured on daily basis throughout three-week rehabilitation and the average values were calculated.

Statistical data analysis was carried out by means of SPSS 17.0 software. The results were shown either as arithmetic mean and standard deviation $(\mathrm{X} \pm \mathrm{SD})$ or as absolute value and percentage. Student's t-test was used for testing parameter values. The value of $p<0.05$ was accepted as statistically significant. Non-parametric values were tested by $\chi^{2}$-test. Spearman's rank correlation coefficient was used for assessing statistical significance of correlation. Multivariate regression analysis was carried out with the aim of defining independent predictors for not taking statin.

\section{Results}

In terms of gender structure, there were $68.6 \%$ of male and $31.4 \%$ of female patients (Table 1). Majority of patients completed four-year secondary education -284 (49.6\%), while 171 (29.8\%) patients completed elementary education. The total of $86(15 \%)$ patients had higher education degree and $32(5.6 \%)$ patients had no formal education whatsoever. On average, the patients who did not use statins had lower education degree level $(\mathrm{p}<0.01$; Table 1). The subgroup of patients who did not take statin had higher values of total cholesterol, LDL cholesterol $(\mathrm{p}<0.001)$ and triglycerides $(\mathrm{p}<0.01)$. Moreover, general obesity and abdominal obesity $(\mathrm{p}<0.01)$, physical inactivity $(p<0.01)$ and presence of positive heredity for cardiovascular disease $(\mathrm{p}<0.001)$ were quite present among the members of this subgroup. Additionally, patients from this subgroup had more prescribed medications a day as compared to the subgroup which took statins $(8.2 \pm 2.8$ vs. $7.5 \pm 2.8 ; \mathrm{p}<0.05)$.

Multivariate regression analysis included the following parameters: age, gender, education, income, number of comorbidities, recurrent cardiovascular events, time which elapsed from cardiovascular event, total number of medications taken per day. This statistical method defined female gender (coefficient $\beta=0.152$ ), monthly income (coefficient $\beta=-0.162$ ) and comorbidities (coefficient $\beta=$ 0.129 ) as independent factors for not taking statin in this model (for model: $\mathrm{R}=0.291, \mathrm{R}^{2}=0.85$, adjusted $\mathrm{R}^{2} 0.80$, std. error of the estimate $0.36151 ; \mathrm{p}<0.001$ ).

Table 2 shows the distribution of previously formulated answers in terms of the reasons for not taking statin.

In terms of percentage of patients who used some kind of reminder for taking medication (medication dosette, notes, telephone, another person, etc.), the figures were not much different in group which did not take medications as compared to the group which took medications (30.6\% vs. $34.9 \%$ )

When asked to state the main reason for not taking statin, majority of patients - 45 wrote that the main problem was lack of financial funds (Figure 1), 21 patients wrote that their laboratory results of lipid status were normal, 11 patients had new health problems, 10 patients were not prescribed statin until the period of rehabilitation, physician discontinued therapy in 7 patients, while 4 patients wrote down that the main reason for not taking statin was their forgetfulness and negli-

Table 1 Clinical features of all patients and differences in parameters in patients who take statin as compared to patients who do not take statin.

\begin{tabular}{lccc}
\hline Parameters & All patients & $\begin{array}{c}\text { Patients } \\
\text { who take statin }\end{array}$ & $\begin{array}{c}\text { Patients } \\
\text { who do not take statin }\end{array}$ \\
\hline Gender m/f & & $346 / 129$ & $47 / 51^{* * *}$ \\
Age & $393 / 180$ & $60.3 \pm 9.0$ & $60.5 \pm 10.1$ \\
Education (level)* & $60.3 \pm 9.9$ & $2.9 \pm 0.9$ & $2.6 \pm 0.6^{* *}$ \\
Smoking (n/\%) & $2.8 \pm 0.9$ & $252(46.9 \%)$ & $36(36.7 \%)$ \\
Waist (cm) & $288(50.2 \%)$ & $98.4 \pm 12.2$ & $101.8 \pm 9.4^{*}$ \\
Abdominal obesity (n/\%) & $99.0 \pm 11.8$ & $228(48.0 \%)$ & $64(65.3 \%)^{* * *}$ \\
Body mass index (g/m $\left.{ }^{2}\right)$ & $292(50.9 \%)$ & $27.3 \pm 4.2$ & $28.6 \pm 4.4^{* *}$ \\
Total cholesterol (mmol/L) & $27.5 \pm 4.2$ & $4.4 \pm 1.0$ & $4.9 \pm 1.1^{* * *}$ \\
HDL cholesterol (mmol/L) & $4.4 \pm 1.1$ & $1.1 \pm 0.4$ & $1.0 \pm 0.2$ \\
LDL cholesterol (mmol/L) & $1.1 \pm 0.3$ & $2.5 \pm 0.9$ & $2.9 \pm 0.9^{* * *}$ \\
Triglyceride (mmol/L) & $2.5 \pm 0.9$ & $1.9 \pm 0.9$ & $2.1 \pm 1.5^{* *}$ \\
Glycemia (mmol/L) & $1.8 \pm 1.0$ & $6.1 \pm 2.1$ & $6.0 \pm 1.7$ \\
Systolic pressure (mmHg) & $6.1 \pm 2.1$ & $123.8 \pm 14.4$ & $126.4 \pm 21.3$ \\
Diastolic pressure (mmHg) & $124.2 \pm 15.8$ & $77.6 \pm 6.1$ & $78.2 \pm 6.7$ \\
Heredity (n/\%) & $77.7 \pm 6.2$ & $273(57.5 \%)$ & $81(82.6 \%)^{* * *}$ \\
Physical inactivity $(\mathrm{n} / \%)$ & $354(61.8 \%)$ & $110(23.1 \%)$ & $38(38.8 \%)^{* *}$ \\
\hline
\end{tabular}

$\mathrm{m}$ - male; f - female; HDL - high-density lipoprotein; LDL - low-density lipoprotein 
Table 2 Distribution of potential reasons for not taking statin

\begin{tabular}{lc}
\hline Potential reason for not taking the medication & Number of patients (n / \%) \\
\hline Physician did not recommend the medication upon hospital discharge & $28(28.6)$ \\
Medication was not prescribed by primary health protection physician & $41(41.8)$ \\
Low monthly income in the family & $71(72.4)$ \\
I forgot to take the medication (always or sometimes) & $39(40.1)$ \\
Lack of information regarding medication benefits & $32(32.6)$ \\
Medication was discontinued when lipid status was within normal limits & $36(36.7)$ \\
I am cured (by-pass or stent), I do not need medication & $12(12.2)$ \\
I am afraid that "medication does not go well with other medications" & $82(83.7)$ \\
I am afraid that I may damage other organs & $38(38.8)$ \\
Medication caused new health issues & $14(14.3)$ \\
I was worried (frightened) when I read medication instruction & $0(0.0)$ \\
I cannot purchase the medication (no specific reason) & $58(59.1)$ \\
I decided to stop taking the medication on my own & $89(90.8)$ \\
\hline
\end{tabular}

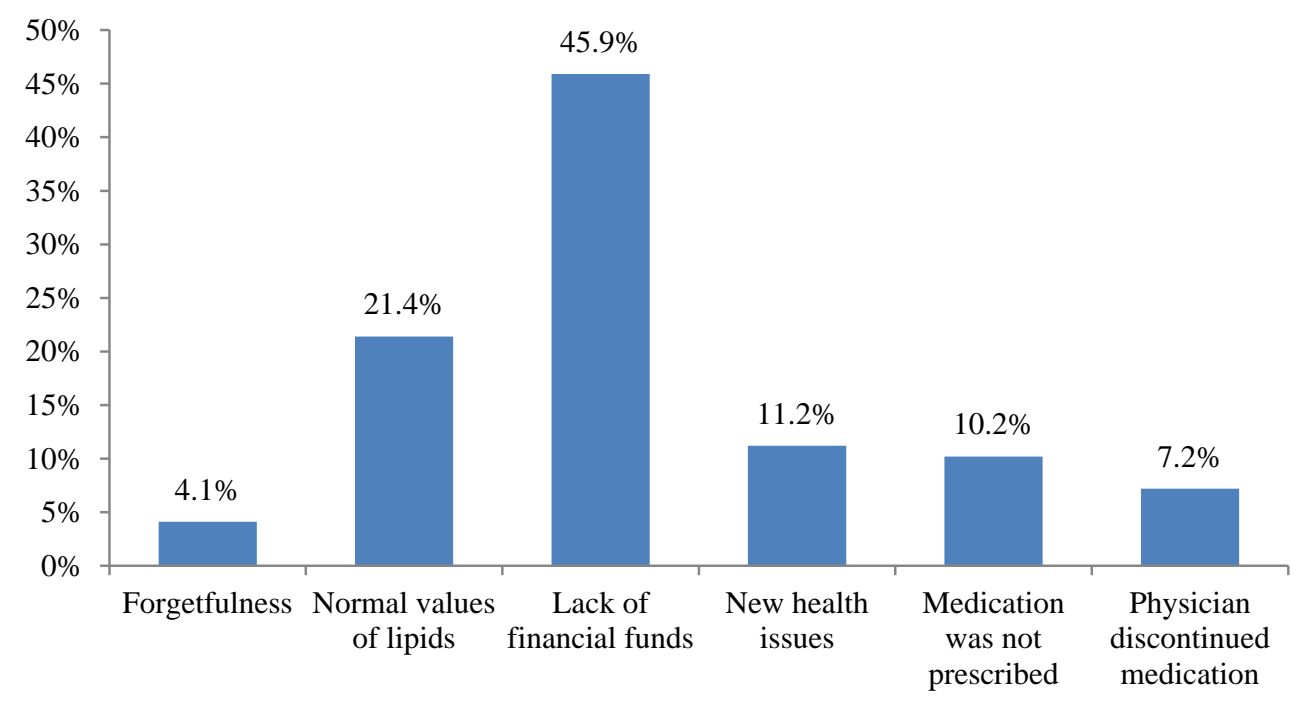

Fig. 1 Distribution of main reasons for not taking statin, according to patient's statements

gence. In terms of health issues that the patients connected with the use of statin, $32(6.7 \%)$ patients reported minor health issues (as compared to subgroup that did not take statins $\mathrm{p}<0.05$ ), but they still took the prescribed therapy.

\section{Discussion}

Nowadays, there is a tendency for standardizing the definition of adherence in medical therapy with the aim of using and comparing data in electronic database, which would make an exceptional base for conducting future meta-analysis [7]. This study could not determine Proportion of Days Covered (PDC) and/or Medication Possession Ratio (MPR), having in mind that the patients have been referred to rehabilitation from various regions in Serbia. However, we could detect the patients who do not take statins by simple physical inspection of the medications which they have brought at the first examination. We have found that $17.1 \%$ of the patients stopped taking statins after 3.2 months from the last acute cardiovascular event. The patients have filled out a questionnaire which contained the reasons for not taking statins and could write down the main reason for not taking the medication.

It is well known that high blood cholesterol levels are associated with an increased risk of CVD events and deaths, and the use of statins is associated with a significant reduction in that risk [8]. In the modellingbased study of Yang et al., under the 2013 Guidelines for primary prevention of atherosclerotic cardiovascular disease using statin therapy, up to $12.6 \%$ of total annual atherosclerotic cardiovascular disease deaths could be prevented among adults aged 40-75 who are eligible for statin treatment $[9,10]$. However, these prevented deaths could be accompanied by additional cases of diabetes or myopathy. The study by Xie et al. pointed out the importance of adherence to statin therapy in prevention of major adverse cardiac events (MACE) and thus clinicians should aim to achieve higher dosage, if tolerable [11]. Therefore, nonadherence represented an important therapy problem in clinical medicine, especially in terms of implementation of guideline for good clinical practice. The study by Kumbhani et al. proved low ad- 
herence to medications for secondary prevention (48.2\%) after one-year follow-up of arteriosclerosis (37154 patients), as well as high frequency of unwanted cardiovascular events and mortality in nonadherent group [12]. Bansilas et al. demonstrated that frequency of large unwanted cardiovascular events was significantly lower in cases of total adherence (statins and ACE inhibitors), i.e. $\geq 80 \%$ of days covered by therapy, as compared to partial adherence and nonadherence in patients after myocardial infarction [13]. They found that adherence had to be minimum $40 \%$ for longer period of time so that the difference in disease outcome would be noticeable. Furthermore, after examining a group of patients with diabetes, Ruokoniemi et al. stated that reduced MACE incidence was observed in patients without any documented cardiovascular disease at statin initiation odds ratio (OR) 0.87 (95\% CI $0.78-0.96)$ overall and OR 0.80 (95\% CI 0.66-0.97) for those who were subjected to 5-year or longer follow-up [14]. The authors concluded that good adherence to statins (the proportion of days covered $\geq 80 \%$ ) predicted reduced incidence of MACEs, irrespective of the presence of coronary heart diseases at statin initiation.

In order to increase the level of adherence, which is based on forgetfulness and/or negligence, the literature has offered various types of reminders for taking medications. For instance, daily alarms combined with individual or partner feedback improved statin medication adherence [15]. The total of $40 \%$ of the members of our group stated that they occasionally forgot to take the medication. However, this was not a crucial reason for not taking statin, as forgetfulness was equally present in both subgroups.

Based on research results, Latry et al. concluded that adherence to statins was poor, but better for those patients with higher number of associated cardiovascular risk factors [16]. The results confirmed that long-term drug treatments were a difficult challenge, particularly for patients who could not see the benefit or felt that they were at risk. Patients at high risk for cardiovascular events were suboptimally dosed with statins, had high rates of discontinuation and low rates of adherence. De- spite the use of statin therapy, atherosclerotic cardiovascular disease-related inpatient visit rates were high, particularly among those patients at highest risk because of a recent acute coronary syndrome hospitalization [17]. In terms of our study, an independent factor for not taking statins was large number of comorbidities. Additionally, larger number of prescribed medications that patients had to take significantly reduced adherence to statins. Larger number of comorbidities and prescribed medications required significant financial funds in home budget, which reduced adherence to statins. Our study has proved that one of independent factors for not taking statins is low family income, which has a logical correlation with two previously stated reasons. We have not noticed differences in answer distribution in tested subgroups when it comes to statin significance, prolongation of life by the use of statins, normalization of lipid parameter level, organ damage due to statins or fear from medication interaction. Therefore, our research has demonstrated that reasons for not taking statins are not subjective, but are objective, i.e. they correlate with education degree, financial conditions, the fact that physician has not prescribed medication or discontinued medication. This points to the significance of the problem of adherence to statin in therapy. Unfortunately, this problem goes way beyond medical profession.

\section{Conclusion}

On average, $17.1 \%$ of patients in Serbia have stopped taking statins 3.2 months after acute coronary event. Low adherence to statins was closely correlated with female gender, low home budget and larger number of comorbidities.

Acknowledgements: We hereby thank Jasmina Nedeljkovic for providing language and proofreading assistance during the creation of this paper. We also thank the students of the Faculty of Medicine Niš, Milan Ivanović and Aleksandar Đorđević, who participated in conducting the survey and preparing the parameters for statistical processing.

\section{References}

1. The Scandinavian Simvastatin Survival Study Investigators. Randomised trials of cholesterol lowering in 4444 patients with coronary heart disease: the Scandinavian Simvastatin Survival Study (4S). Lancet 1994; 344:1383-1389.

2. Van de Werf F, Bax J, Betriu A, Blomstrom-Lundqvist C, Crea F, Falk V, et al. Management of acute myocardial infarction in patients presenting with persistent ST-segment elevation. The Task Force on the management of ST-segment elevation acute myocardial infarction of the Europen Society of Cardiology. Eur Heart J, 2008; 29:2909-2945.

3. Reiner Z, De Backer G, Fras Z, et al. Lipid lowering drugs therapy in patients with coronary heart disease from 24 European countries - findings from the EUROASPIRE IV study. Atherosclerosis 2016; 246:243-250.

4. Halvorsen S, Jortveit J, Hasvold P, Thuresson M, Oie E. Initiation of and long-term adherence to secondary preventive drugs after acute myocardial infarction. BMC Cardiovasc Disord 2016; 16:115.

5. Mann DM, WoodwardM, Muntner P, Falzon L, Kronish I. Predictors of nonadherence to statins: A systematic review and meta-analysis. Ann Pharmacother 2010; 44:1410-1421.

6. Tasic I, Lazarevic G, Kostic S, Djordjevic D, Simonovic D, Rihter $M$, et al. Administration and effect of secondary prevention measures in coronary heart disease patients from Serbia according to gender and cardiometabolic risk. Acta Cardiol 2010; 65(4):407414.

7. Raebel MA, Schmittdiel J, Karter AJ, Konieczny JL, Steiner JF. Standardizing terminology and definitions of medication adherence and persistence in research employing electronic databases. Med Care 2013; 51(803):S11-S21.

8. Stone NJ, Robinson JG, Lichtenstein AH, et al. Treatment of blood cholesterol to reduce atherosclerotic cardiovascular disease risk in adults: synopsis of the 2013 ACC/AHA cholesterol guideline. Ann Intern Med 2014; 160:339-343. 
9. Yang Q, Zhong Y, Gillespie C, et al. Assessing potential population impact of statin treatment for primary prevention of atherosclerotic cardiovascular diseases in the USA: population-based modelling study. BMJ Open 2017; 7:e011684.

10. Goff DC, Jr., Lloyd-Jones DM, Bennett G, et al. 2013 ACC/AHA guideline on the assessment of cardiovascular risk: a report of the American College of Cardiology/American Heart Association Task Force on practice guidelines. Circulation 2014; 129(25 Suppl 2):S49-73.

11. Xie G, Sun Y, Myint PK, et al. Six-month adherence to statin use and subsequent risk of major adverse cardiovascular events (MACE) in patients discharged with acute coronary syndromes. Lipids Health Dis 2017; 16:155-162.

12. Kumbhani DJ, Steg G, Cannon CP, et al. Adherence to secondary prevention medications and four-year outcomes in outpatients with atherosclerosis. Am J Med 2013; 126:693-700.
13. Bansilal S, Castellano JM, Garrdio E, et al. Assessing the impact of medication adherence on long-term cardiovascular outcomees. JACC 2016; 68(8):789-801.

14. Ruokonieml P, Korhonen MJ, Helin-Salmlaara A, et al. Statin adherence and the risk of major coronary events in patients with diabetes: a nested case-control study. Br J Clin Pharmacol 2011; 71(5):766-776.

15. Reddy A, Huseman TL, Canamucio A, et al. Patient and partner feedback reports to improve statin medication adherence: a randomized control trial. J Gen Intern Med 2017; 32(3):256-261.

16. Latry P, Molimard M, Dedieu B, Couffinhal T, Begaud B, MartinLatry K. Adherence with statins in real-life setting is better when associated cardiovascular risk factors increase: a cohort study. BMC Cardiovasc Disord 2011; 11:46.

17. Lin I, Sung J, Sanchez RJ, et al. Patterns of statin use in a real-wold population of patients at high cardiovascular risk. J Manag Care Spec Pharm 2016; 22(6):685-698. 